\title{
Nivel de satisfacción de los pacientes postoperados de traumatismo facial: Un estudio de cohorte de un Hospital Nacional en Perú
}

\author{
Gustavo Sáenz-Poma ${ }^{1}$, Roberto Chávez-Asmat ${ }^{2,3}$, Javier Silva-Valencia ${ }^{3,4}$, \\ Sonia Simón-Romero ${ }^{3,4}$ y Paul Jesús Tejada-Llacsa ${ }^{3,4}$
}

'Cirujano de cabeza, cuello y maxilofacial. Hospital Nacional Dos de Mayo.

Laboratorio de Farmacología Universidad Nacional Mayor de San Marcos. Lima, Perú. ${ }^{3}$ Asociación para el Desarrollo de la Investigación Estudiantil en Ciencias de la Salud (ADIECS-UNMSM). Lima, Perú.

${ }^{4}$ Facultad de Medicina San Fernando, Universidad Nacional Mayor de San Marcos. Lima, Perú.

Recibido el 26 de mayo de 2017, aceptado el 31 de julio de 2017

Correspondencia a: Paul Jesús Tejada Llacsa Av. Gonzales Prada 719 El Carmen, Comas. paultejada24@gmail.com
Satisfaction level of post-operated facial trauma patients. A cohort study of a National Hospital in Peru

Objective: To determine the level of satisfaction of patients who underwent surgery for facial trauma in the Dos de Mayo National Hospital (HNDM). Materials and Methods: Quantitative, cross-sectional analytical study. The population consisted of 36 patients diagnosed with facial trauma who underwent surgery in the Head and Neck service of the HNDM during the period July 2014-February 2015. A Global satisfaction questionnaire, previously validated for this research, was used whose reliability is $r=0.95$ and evaluates aspects of Limitation functional, facial appearance, sexual and physical appearance, negative self- concept and social appearance. The average scores on the Likert scale were compared with the paired Student $t$ test. A p-value $<0.05$ was considered statistically significant. Results: Regarding the overall satisfaction of patients, the number of patients who reported having a high level of satisfaction was significantly higher in the post surgery compared to what was achieved in the pre surgery $(p=0.01)$. In respect of functional limitations, facial appearance, negative self-concept and social appearance $100 \%$ achieved a high level of satisfaction in the post surgery. In the dimension of sexual and physical appearance, $100 \%$ was in the post surgery a medium level of satisfaction, from the low level obtained in the pre surgery. Conclusions: The patients operated after facial trauma in the Dos de Mayo National Hospital have a high level of satisfaction on a functional level, facial appearance, negative self-concept and social appearance. Key words: Facial injuries, patient satisfaction, postoperative period.

\section{Resumen}

Objetivo: Determinar el nivel de satisfacción de los pacientes postoperados de traumatismo facial en el Hospital Nacional Dos de Mayo (HNDM). Material y Método: Estudio cuantitativo, cohorte longitudinal, analítica. La población estuvo constituida por 36 pacientes con diagnóstico de traumatismo facial que se sometieron a cirugía en el servicio de cabeza y cuello del HNDM durante el periodo julio de 2014-febrero de 2015. Se utilizó un cuestionario sobre satisfacción global cuya confiabilidad es $r=0,95$, que evalúa cinco dimensiones: Limitación funcional, apariencia facial, apariencia sexual y corporal, autoconcepto negativo y apariencia social. Los puntajes promedio obtenidos en la escala de Likert fueron comparados con la prueba $\mathrm{T}$ de student pareada. Se consideró un valor $\mathrm{p}<0,05$ como estadísticamente significativo. Resultados: La satisfacción global de los pacientes postcirugía fue alta en comparación con lo alcanzado en la precirugía $(p=0,01)$. En las dimensiones limitación funcional, apariencia facial, autoconcepto negativo y apariencia social el $100 \%$ alcanzó un nivel de satisfacción alto en la postcirugía. En la dimensión de apariencia sexual y corporal, el $100 \%$ tuvo en el periodo postcirugía un nivel de satisfacción medio, respecto al nivel bajo obtenido en la precirugía. Conclusión: Los pacientes postoperados de traumatismo facial en el Hospital Nacional Dos de Mayo presentan un alto nivel de satisfacción a nivel funcional, de apariencia facial, en autoconcepto y de apariencia social.

Palabras clave: Traumatismo facial, nivel de satisfacción, período postoperatorio. 


\section{Introducción}

A nivel mundial, el traumatismo facial causa 300.000 muertes anualmente y más de 8 millones de heridos ${ }^{1,2}$. En Latinoamérica, los traumatismos faciales son más o frecuentes en varones entre 21 y 40 años $^{3}$. En el Perú, existe un similar perfil epidemiológico en cuanto a la edad y el sexo siendo las causas más frecuentes los accidentes de tránsito $(40 \%)$ y las agresiones físicas $(30 \%)^{4,5}$.

Muchos pacientes con traumatismo facial son sometidos a un rechazo social ${ }^{6}$. En general, se acepta que los pacientes con deformidades faciales se ven a sí mismos como impedidos socialmente, con restricciones y carentes de habilidades sociales ${ }^{7}$. Es así que la cirugía correctiva en pacientes con traumatismo facial tiene una gran repercusión en el aspecto físico y psicológico, por lo que no sólo deberá estar orientada a la mejora física y funcional sino de lograr preservar la estética e impactar positivamente en la satisfacción del paciente en áreas como la autoestima y las relaciones interpersonales ${ }^{8}$.

En el Perú, no existen datos sobre la satisfacción en el paciente postoperado de traumatismo facial. La mayoría de estudios publicados se orientan a medir satisfacción del punto de vista de "calidad de atención" o "trato del profesional de salud", dejando de lado la satisfacción del paciente en relación a los resultados de su cirugía. Esto implica abordar aspectos psicológicos y físicos, es decir, entender a la persona bajo un enfoque integral.

Debido a lo expuesto, la presente investigación tuvo como objetivo determinar el nivel de satisfacción de los pacientes postoperados de traumatismo facial en el Hospital Nacional Dos de Mayo durante el año 2015.

\section{Material y Métodos}

Estudio cuantitativo, cohorte longitudinal, analítico. La población estuvo constituida por los pacientes con diagnóstico de traumatismo facial que acuden al Hospital Nacional Dos de Mayo (HNDM) durante el periodo julio de 2014-febrero de 2015.

\section{Criterios de inclusión}

Paciente que brindó su consentimiento informado y en el caso de menores de edad, los que dieron su asentimiento y su apoderado firmara el consentimiento informado.

\section{Criterios de exclusión}

Pacientes con trastorno dismórfico corporal, trastornos psiquiátricos diagnosticados y que tengan limitaciones para comunicarse (sordo, mudo, que no hable español).

\section{Diseño del cuestionario, pilotaje y validación}

El cuestionario fue diseñado y adaptado a nuestra realidad. Para ello fue sometido a validez de contenido y constructo mediante juicio de expertos con 7 profesionales: médicos especialistas en cirugía de cabeza y cuello $(\mathrm{n}=3)$, cirugía plástica $(\mathrm{n}=2)$, psiquiatría $(\mathrm{n}=1)$ y salud pública $(\mathrm{n}=1)$, quienes proporcionaron sus sugerencias y modificaciones respecto a la relevancia del contenido de los ítems y a la adaptación del texto al lenguaje habitualmente utilizado en nuestro país; se aplicó la tabla de concordancia y la prueba binomial $(\mathrm{p}=0,038)$. Posteriormente, se realizó un pilotaje en 8 pacientes, se determinó la confiabilidad del instrumento con la prueba Kuder Richarson, obteniéndose para limitación funcional un $r=0,90$, para apariencia facial un $r=0,87$, apariencia sexual y corporal un $r=0,76$, autoconcepto negativo un $r=0,77$, apariencia social un $\mathrm{r}=0,77$ y como satisfacción global un $\mathrm{r}=0,95$.

Se calculó la correlación de Pearson entre los ítems y la puntuación total de la escala de satisfacción global. Las preguntas de limitación funcional se establecieron a través de los síntomas reportados en las historias clínicas y solo 13 preguntas presentaron valor $r>0,5$. Con respecto a la apariencia facial, social, de autoconcepto negativo, sexual y corporal se consideraron las preguntas de "Derriford Appearance Scale (DAS59)" " presentando un valor $\mathrm{r}>0,5$, solo 28 de las 38 del artículo original. Finalmente el cuestionario sobre satisfacción global consta de 41 preguntas: limitación funcional (13 preguntas), apariencia facial (5 preguntas), apariencia sexual y corporal (7 preguntas), autoconcepto negativo (5 preguntas) y apariencia social (11 preguntas). (Tabla 1).

Las respuestas fueron tipo Likert: nunca (0), rara vez (1), algunas veces (2), casi siempre (3) y siempre (4), los puntajes fueron categorizados en satisfacción global como alto, medio y bajo a través de la escala de Estanones; esta categorización utilizó la comparación cualitativa a través de la Campana de Gauss, usándose la formula $\mathrm{a} / \mathrm{b}=\mathrm{X} \pm 0,75$ (DS) (dónde: $\mathrm{a} / \mathrm{b}=$ valores máximo/mínimo, $\mathrm{X}=$ promedio de puntajes, $\mathrm{DS}=$ desviación estándar), finalmente se estableció puntaje de limitación funcional: Satisfacción alta $(<0,52)$, media $(0,52-1,90)$ y baja $(>1,90)$; para apariencia facial: Satisfacción alta $(<0,44)$, media $(0,44-2,24)$ y baja $(>2,24)$; para apariencia sexual y corporal: Satisfacción alta $(<0,39)$, media $(0,39-2,36)$ y baja $(>2,36)$; para 
Tabla 1. Preguntas que incluye cada dimensión del cuestionario

\begin{tabular}{|c|c|}
\hline $\begin{array}{l}\text { Limitación } \\
\text { funcional: } \\
13 \text { preguntas }\end{array}$ & $\begin{array}{l}\text { ¿Ha presentado dolor o cansancio en los músculos de la cara? } \\
\text { ¿Ha presentado dolores de cabeza a repetición? } \\
\text { ¿Ha presentado dolores o sensación de oídos llenos o tapados? } \\
\text { ¿Ha presentado visión borrosa o doble? } \\
\text { ¿Ha presentado dolor referido a los hombros, espalda o brazo? } \\
\text { ¿Tiene dificultad para dormir? } \\
\text { ¿Ha tenido dolor o molestias para masticar alimentos? } \\
\text { ¿Ha tenido dificultad para abrir o cerrar la boca? } \\
\text { ¿Ha presentado ruidos al abrir o cerrar la mandíbula? } \\
\text { ¿Ha tenido problemas pronunciando alguna palabra? } \\
\text { ¿La gente ha malentendido algunas de tus palabras? } \\
\text { ¿Has sentido que tu sensación de sabor ha empeorado? } \\
\text { ¿Has sentido que hay menos sabor en tus alimentos? }\end{array}$ \\
\hline $\begin{array}{l}\text { Apariencia } \\
\text { sexual y } \\
\text { corporal: } \\
7 \text { preguntas }\end{array}$ & $\begin{array}{l}\text { Evita desvestirse en frente de su pareja } \\
\text { Evita vestuarios comunales } \\
\text { Piensa negativamente sobre la vida sexual } \\
\text { Piensa negativamente sobre el matrimonio } \\
\text { Se angustia cuando va a la playa } \\
\text { Se angustia de no poder usar ropa favoritas } \\
\text { Se angustia de ser incapaz de mirarse en el espejo }\end{array}$ \\
\hline $\begin{array}{l}\text { Apariencia } \\
\text { facial: } \\
5 \text { preguntas }\end{array}$ & $\begin{array}{l}\text { Siente la necesidad de usar una gorra/pañoleta para salir a calle } \\
\text { Evita que su pelo esté mojado } \\
\text { Se angustia de no poder cambiar de peinado } \\
\text { Se angustia de no poder responder cuando llaman a la puerta } \\
\text { Se angustia de no poder salir en días con mucho viento }\end{array}$ \\
\hline $\begin{array}{l}\text { Autoconcepto } \\
\text { negativo: } \\
5 \text { preguntas }\end{array}$ & $\begin{array}{l}\text { ¿Usted siente confianza en sí mismo? } \\
\text { ¿Usted se siente seguro de sí mismo? } \\
\text { ¿Usted se siente alegre? } \\
\text { ¿Usted se siente normal? } \\
\text { ¿Usted se siente cómo hombre/mujer? }\end{array}$ \\
\hline $\begin{array}{l}\text { Apariencia } \\
\text { social: } \\
11 \text { preguntas }\end{array}$ & $\begin{array}{l}\text { Evita a los niños en la calle } \\
\text { Tiene dificultad para hacer amigos } \\
\text { Evita la escuela/universidad/trabajo } \\
\text { Evita salir de casa } \\
\text { Siente que es una vergüenza para amigos/familia } \\
\text { Se siente rechazado } \\
\text { Se siente inútil } \\
\text { Siente angustia cuando va a la escuela/trabajo } \\
\text { Siente dificultad para hacer su actividad diaria } \\
\text { Se angustia cuando viaja en el transporte público } \\
\text { Se angustia al no poder ir a eventos sociales }\end{array}$ \\
\hline
\end{tabular}

apariencia autoconcepto negativo: Satisfacción alta $(>3,40)$, media $(1,53-3,40)$ y baja $(<1,53)$; para apariencia social: Satisfacción alta $(<0,36)$, media $(0,36-1,81)$ y baja $(>1,81)$; para satisfacción global: alta $(<0,90)$, media $(0,90-1,82)$ y baja $(>1,82)$.

Se realizaron los trámites administrativos mediante un oficio de la unidad de postgrado Univer- sidad Nacional Mayor de San Marcos (UNMSM) dirigido a HNDM a fin de obtener la autorización para ejecutar el estudio. Luego de haber sido aceptado se realizó las coordinaciones pertinentes con la Jefatura del Servicio de Cirugía de Cabeza y Cuello así como con el personal de salud del Servicio para darle a conocer los fines del estudio y establecer el cronograma de inicio de la recolección de datos durante los meses de julio de 2014-febrero de 2015. La aplicación del instrumento tuvo una duración promedio de 25 a $30 \mathrm{~min}$, previa autorización del paciente (consentimiento/asentimiento informado).

\section{Período preoperatorio}

El cuestionario fue administrado en la última visita al consultorio previa cirugía o durante la hospitalización días previos a la intervención quirúrgica programada.

\section{Período postoperatorio}

El cuestionario fue administrado después de la visita postoperatoria a los 3-4 meses. Se consideró este tiempo para esperar la cicatrización temprana y para permitir que el paciente pueda evaluar adecuadamente los cambios ocurridos en su imagen corporal.

Los puntajes en la escala de Likert fueron expresados en promedio \pm desviación estándar y comparada con la prueba $T$ de student pareada. Se utilizó el paquete estadístico SPSS vs 18, considerando significativo un $\mathrm{p}<0,05$.

Dentro de las consideraciones éticas, se tomó en cuenta las coordinaciones con la Jefatura del Servicio de Cabeza y Cuello-HNDM y el consentimiento/ asentimiento informado previa información verbal y escrita que se otorgó al apoderado del adolescente si era menor de edad o del mismo si tenía/era mayor de 18 años. Se contó con la aprobación del comité de ética del HNDM en Lima, Perú. Número: 076-2014-CEIB-AI-OACDI-HNDM.

\section{Resultados}

De un total de 41 paciente que iniciaron el estudio fueron excluidos 3 al no ser posible una comunicación comprensiva debido al idioma (quechua) y 2 pacientes por no acudir al control postoperatorio. Todos fueron traumatismo pan faciales, 23 casos con fractura mandibular y fractura de tercio medio facial (fractura del complejo cigomático maxilar), 8 con fractura de la pared anterior del seno frontal y orbitaria, finalmente 5 con fractura frontal y del tercio medio facial (fractura nasoorbitoetmoidal). Referente a la etiología del traumatismo, 25 fueron 
Tabla 2. Características sociodemográficas de los pacientes postoperados de traumatismo facial en el Hospital Nacional Dos de Mayo, 2015

\begin{tabular}{|lrc|}
\hline Características & n & \% \\
Sexo & & \\
Femenino & 11 & 30,6 \\
Masculino & 25 & 69,4 \\
Edad (años) & & \\
$\quad<20$ & 5 & 14 \\
$20-29$ & 5 & 14 \\
$30-39$ & 15 & 41,4 \\
$>40$ & 11 & 30,6 \\
Estado civil & & \\
Soltero & 20 & 55,4 \\
Conviviente & 6 & 16,6 \\
Casado & 5 & 14 \\
Separado & 5 & 14 \\
Grado de instrucción & & \\
Primaria C & 0 & 0 \\
Primaria I & 0 & 0 \\
Secundaria C & 5 & 14 \\
Secundaria I & 10 & 28 \\
Superior C & 11 & 30 \\
Superior I & 10 & 28 \\
Analfabeto & 0 & 0 \\
Condición laboral & & \\
Independiente & 11 & 30 \\
Dependiente & 15 & 42 \\
Su casa & 0 & \\
Otros & 10 & \\
\hline
\end{tabular}

accidente de tránsito, 9 accidente laboral y 2 por arma de fuego.

En relación a las características sociodemográficas tenemos que el 69,4\% (25) fueron varones, el 41,4\% tenía entre 30 a 39 años, siendo la edad mínima de 15 años y la máxima de 46 años (Tabla 2).

En relación a las 5 dimensiones evaluadas, todas ellas tuvieron un cambio positivo según la escala evaluada, siendo estas diferencias estadísticamente significativas y obteniéndose una satisfacción alta en 3 dimensiones (limitación funcional, apariencia facial y autoconcepto negativos) (Tabla 3).

Tabla 3. Nivel de satisfacción global de los pacientes postoperados de traumatismo facial en el HNDM, 2015

\begin{tabular}{|c|c|c|c|c|c|}
\hline \multirow{3}{*}{$\begin{array}{l}\text { Dimensiones } \\
\text { Satisfacción global }\end{array}$} & \multicolumn{4}{|c|}{ Nivel de satisfacción* } & \multirow[b]{2}{*}{$\mathbf{p}^{* *}$} \\
\hline & \multicolumn{2}{|c|}{$\begin{array}{l}\text { Precirugía } \\
\quad(n=36)\end{array}$} & \multicolumn{2}{|c|}{$\begin{array}{c}\text { Postcirugía } \\
(\mathrm{n}=\mathbf{3 6})\end{array}$} & \\
\hline & & & & & \\
\hline Limitación funcional & $1,80 \pm 0,95$ & $\mathbf{M}$ & $0,51 \pm 0,35$ & $\mathbf{A}$ & 0,01 \\
\hline Apariencia facial & $2,20 \pm 1,05$ & $\mathbf{M}$ & $0,42 \pm 0,50$ & $\mathbf{A}$ & 0,01 \\
\hline Apariencia sexual y corporal & $2,37 \pm 1,33$ & B & $0,59 \pm 0,50$ & $\mathbf{M}$ & 0,01 \\
\hline Autoconcepto negativo & $1,77 \pm 1,12$ & $\mathbf{M}$ & $3,42 \pm 0,43$ & $\mathbf{A}$ & 0,01 \\
\hline Apariencia social & $1,82 \pm 1,04$ & $\mathbf{B}$ & $0,55 \pm 0,35$ & $\mathbf{M}$ & 0,01 \\
\hline Total & $1,73 \pm 0,60$ & $\mathbf{M}$ & $0,46 \pm 0,18$ & $\mathbf{A}$ & 0,01 \\
\hline
\end{tabular}

*Puntaje de satisfacción expresado en Promedio \pm Desviación estándar. Escala de Estanones Alto (A): <0,90, Moderado (M): 0,90 - 1,82, Bajo (B): > 1,82. **Prueba $t$ student pareada.

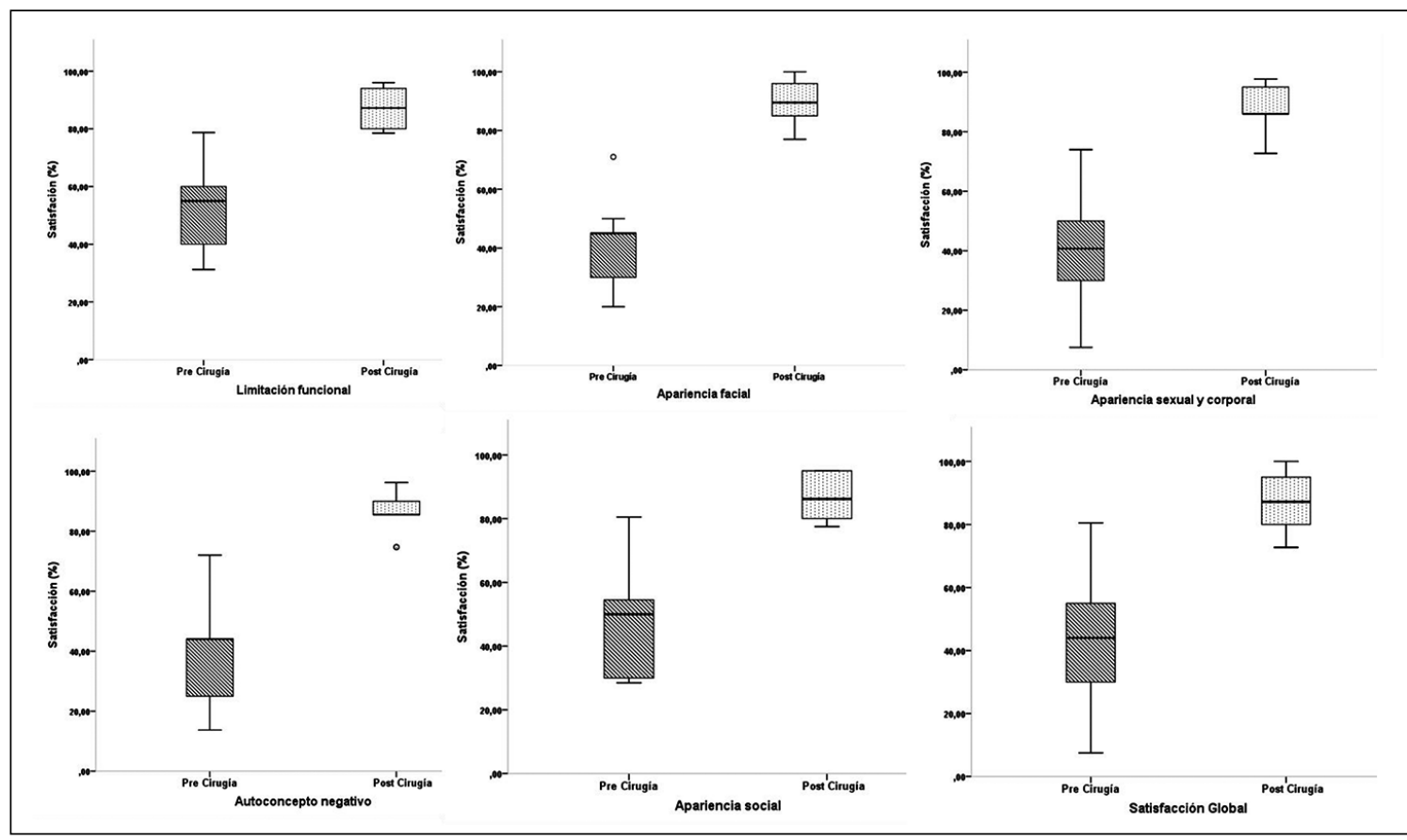

Figura 1. Nivel de satisfacción por dimensiones y global de los pacientes postoperados de traumatismo facial en el HNDM, 2015. 


\section{ARTíCULO DE INVESTIGACIÓN}

Se estima que para la población antes de la cirugía, el nivel satisfacción total esté entre bajo y moderado, y luego de la intervención, el nivel de satisfacción total estimado sería alto, siendo esta diferencia estadísticamente significativa $(\mathrm{p}=0,01)$

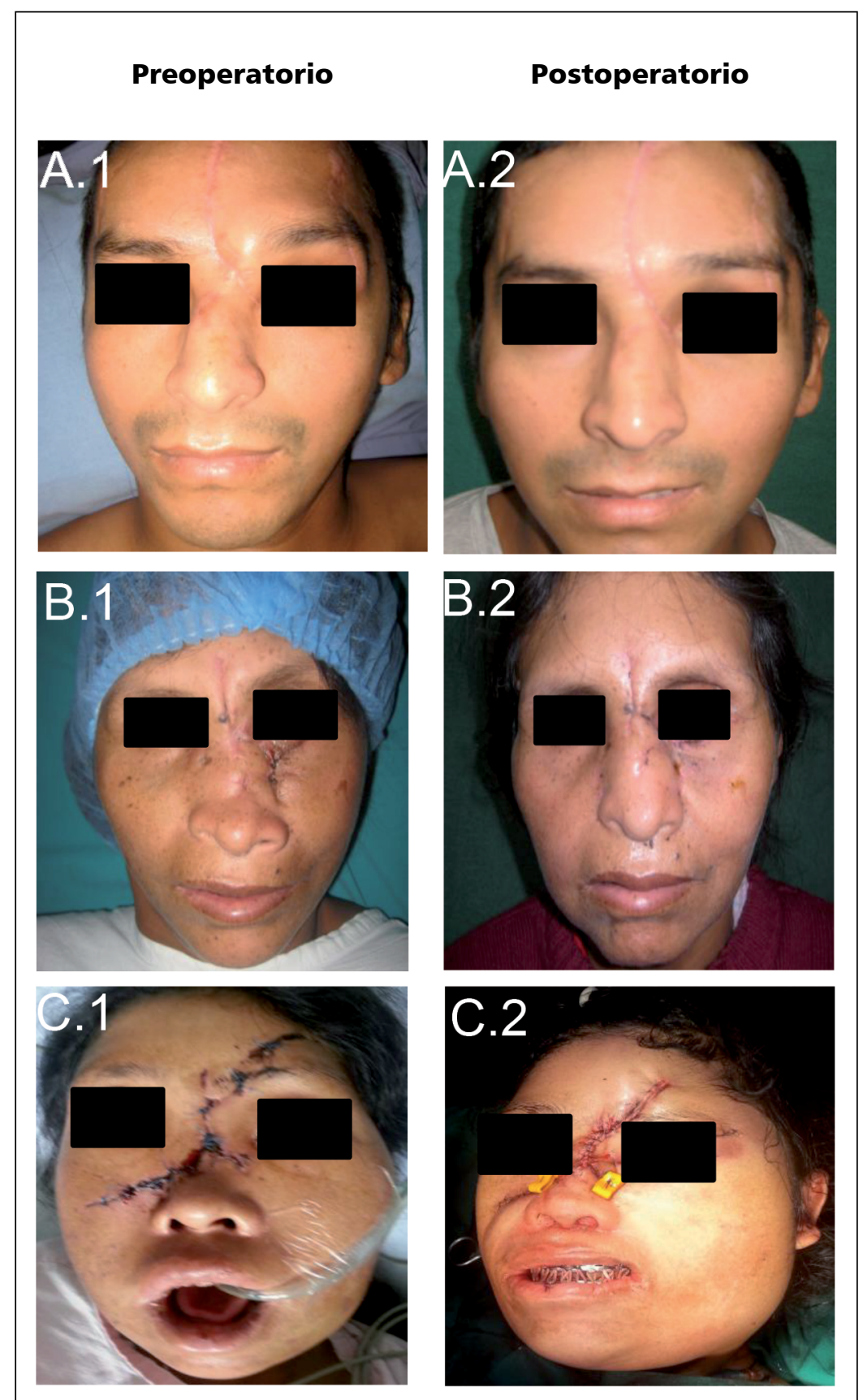

Figura 2. Antes y después de la cirugía. A.1 y A.2: Paciente que obtuvo el puntaje de alta satisfacción. B.1 y B.2: Paciente que obtuvo el puntaje de moderada satisfacción. C.1 y C.2: Paciente que obtuvo el puntaje de baja satisfacción.

\section{Discusión}

La satisfacción global postcirugía logró un nivel alto, en comparación a la precirugía en la que presentaron un nivel de satisfacción medio. La satisfacción de un paciente postoperado de traumatismo maxilofacial estudiado desde distintas dimensiones (limitación funcional, apariencia facial, apariencia sexual y corporal, autoconcepto y apariencia social) no ha sido encontrada en la literatura. Se ha realizado estudios de seguimiento en cuanto a la depresión, ansiedad y estrés en pacientes que sufrieron trauma maxilofacial como lo reporta Pokhrel ${ }^{10}$ donde encuentra que en el seguimiento los niveles de ansiedad, depresión y estrés disminuyen tras el tratamiento quirúrgico, tal como en el caso de depresión severa de un $16,3 \%$ a un $10,9 \%$, ansiedad severa de un $15,2 \%$ a un $11,2 \%$ y el estrés severo de $1,08 \%$ a $0 \%$.

En las 5 dimensiones evaluadas (limitación funcional, apariencia facial, apariencia sexual y corporal, autoconcepto y apariencia social) se encontraron resultados favorables. La dimensión limitación funcional es una de las principales ya que abarca las grandes preocupaciones que conlleva una cirugía reconstructiva o reparadora: mayor o menor facilidad para desempeñar actividades como masticación, higiene oral, entre otras. Los resultados fueron similares a otras investigaciones, tal como lo reporta Souza et $\mathrm{al}^{11}$, donde encuentra una satisfacción estética y de obstrucción respiratoria en $85 \%$ en pacientes postoperados de fracturas nasales; en el presenta trabajo, antes de la intervención, el 30,9\% de pacientes manifestaban valoraciones desfavorables acerca de su limitación funcional y solo el $11,8 \%$ persistió con ese juicio luego de la cirugía.

La dimensión autoconcepto negativo es considerada tan relevante como los problemas funcionales" ${ }^{2}$. En nuestro estudio el 100\% tuvo en la postcirugía un nivel de satisfacción alto, respecto a la precirugía. Esta dimensión en otros estudios fue evaluada como malestar psicológico y en el estudio de Espinosa et $\mathrm{al}^{13}$ reportó una disminución de $80 \%$ en la postcirugía respecto a la pre cirugía, lo cual se tradujo en estados de sentirse mejor consigo mismos y en su actitud hacia la vida.

Referente a la apariencia social, estudios anteriores como de Sadek H y Salem G. ${ }^{14}$ reportaron una mejoría de $58 \%$ en la aceptación social; Espinosa et $\mathrm{al}^{13}$ reportó una mejoría de $85 \%$ en la capacidad para relacionarse con otras personas.

El $69,4 \%$ de los pacientes en nuestro estudio fueron varones. En la literatura se puede encontrar investigaciones de accidentes maxilofaciales donde 
predominan tanto pacientes varones (como la tasa reportada por Hachl O et al $^{15}$ : $11,8 / 1$ o por Eggensperger et al: $41 / 1)$ como pacientes mujeres $(69 \%$ en la investigación de Jill L. Hessler ${ }^{17}$ ). Por otro lado, es importante mencionar que no se ha evidenciado que el factor sexo contribuya a predecir o modificar el grado de satisfacción postoperatoria ${ }^{17}$.

La edad, el grado de instrucción y el estado civil son reconocidos como factores que influyen en el grado de satisfacción del paciente. La edad es un factor importante ya que se ha evidenciado que una mayor edad se asocia significativamente con mayores puntuaciones de satisfacción postoperatoria $(p=0,01)^{17}$. En nuestro estudio la mayor cantidad de pacientes estuvo en el rango de 30-39 años a diferencia de otro estudio donde la media de edad fue de 53 años ${ }^{17}$, sin embargo, estos obtuvieron resultados de satisfacción postoperatoria similares a los nuestros.

Hoy en día el estado de salud y la calidad relacionada con la salud es crítico y debe considerarse durante la evaluación de los resultados de cualquier cirugía. Es vital para la mejoría de la atención del paciente entender que la satisfacción con los resultados del tratamiento está íntimamente ligada con el impacto psicológico que este procedimiento representa.

En conclusión, la satisfacción global de los pacientes postcirugía fue alta, donde, tres dimensiones (limitación funcional, apariencia facial, autoconcep- to) fueron las que lograron un nivel de satisfacción alto.

\section{Responsabilidades éticas}

Protección de personas y animales. Los autores declaran que para esta investigación no se han realizado experimentos en seres humanos ni en animales.

Confidencialidad de los datos. Los autores declaran que han seguido los protocolos de su centro de trabajo sobre la publicación de datos de pacientes.

Derecho a la privacidad y consentimiento informado. Los autores han obtenido el consentimiento informado de los pacientes y/o sujetos referidos en el artículo. Este documento obra en poder del autor de correspondencia.

\section{Agradecimientos}

Al Servicio de Cirugía de Cabeza y Cuello del Hospital Dos de Mayo por las facilidades y los permisos para la realización de la presente investigación.

\section{Conflicto de intereses}

Los autores declaran no tener ningún conflicto de intereses.

\section{Bibliografía}

1. Marques L, Cavalcante GMS, Lima MMSM, Madruga RCR, Ramos-Jorge ML, D'Avila S. Prevalence of facial trauma and associated factors in victims of road traffic accidents. Am J Emerg Med. 2014;32:1382-1386.

2. Lapostolle A, Gadegbeku B, Ndiaye A, Amoros E, Chiron M, Spira A, et al. The burden of road traffic accidents in a French Departement: the description of the injuries and recent changes. BMC Public Health. 2009;9:386.

3. Brasileiro BF, Passeri LA. Epidemiological analysis of maxillofacial fractures in Brazil: A 5-year prospective study. Oral Surgery, Oral Med Oral Pathol Oral Radiol Endodontology. 2006;102:2834.

4. Avello Canisto F. Fracturas del tercio medio facial: experiencia en el Hospital Nacional Dos de Mayo, 1999-2009. An la Fac Med. 2013;74:123-128.
5. Molina Sotomayor H. Prevalencia de traumatismos de la región maxilofacial en pacientes atendidos en dos hospitales de Lima durante el periodo 20002006. 2007.

6. Phillips C, Griffin T, Bennett E. Perception of facial attractiveness by patients, peers, and professionals. Int J Adult Orthodon Orthognath Surg. 1995;10:127-135.

7. Bertolini F, Russo V, Sansebastiano G. Pre- and postsurgical psycho- emotional aspects of the orthognathic surgery patient. Int J Adult Orthodon Orthognath Surg. 2000;15:16-23.

8. Juric M, Novakovic J, Carapina M, Kneievic E. Treatment cost of patients with maxillofacial fractures at the University Hospital in Mostar 2002-2006. Coll Antropo. 2010;34 Suppl 1:199-203.

9. Harris DL, Carr AT. The Derriford Appearance Scale (DAS59): a new psychometric scale for the evaluation of patients with disfigurements and aesthetic problems of appearance. Br J Plast Surg. 2001;54:216-222.

10. Pokhrel PK, Baliga M, Amirthraj A, Mehar H. Assessing psychological consequences following maxillofacial trauma using DASS scale - our experience. Asian J Med Sci. 2016;7:8589.

11. Souza DPE, Filho FN, Temprano AB, Guevara HAG, Leandro LFL. Estudio epidemiológico de fracturas nasales en hospitales particulares de Sao Paulo, Brasil. Rev Española Cir Oral y Maxilofac. 2014; 36: 149-155.

12. Ostler S, Kiyak HA. Treatment expectations versus outcomes among orthognathic surgery patients. Int J Adult Orthodon Orthognath Surg. 1991;6:247255.

13. Espinosa N, Montoya S, Orozco J, Valencia A, Zuluaga AM. Adaptación funcional y psicosocial de cirugía de articulación temporomandibular. Rev CES Odontol. 2001;14:19-23. 


\section{ARTíCULO DE INVESTIGACIÓN}

14. Sadek H, Salem G. Psychological aspects of orthognathic surgery and its effect on quality of life in Egyptian patients. East Mediterr Heal J. 2007;13:150-159.

15. Hachl O, Tuli T, Schwabegger A, Gassner R. Maxillofacial trauma due to work-related accidents. Int J Oral Maxillofac Surg. 2002;31:90-93.

16. Eggensperger NM, Danz J, Heinz Z, lizuka T. Occupational maxillofacial fractures: A 3-year survey in Central Switzerland. J Oral Maxillofac Surg.
2006;64:270-276.

17. Hessler JL, Moyer CA, Kim JC, Baker SR, Moyer JS. Predictors of satisfaction with facial plastic surgery: results of a prospective study. Arch Facial Plast Surg. 2010;12:192-196. 